\title{
Approximate expressions for the period of a simple pendulum using Taylor series expansion
}

\author{
Augusto Beléndez ${ }^{1,2}$, Enrique Arribas ${ }^{3}$, Andrés Márquez ${ }^{1,2}$, Manuel Ortuño ${ }^{1,2}$ and \\ Sergi Gallego ${ }^{1,2}$
}

(1) Departamento de Física, Ingeniería de Sistemas y Teoría de la Señal.

Universidad de Alicante. Apartado 99. E-03080 Alicante. SPAIN

(2) Instituto Universitario de Física Aplicada a las Ciencias y las Tecnologías.

Universidad de Alicante. Apartado 99. E-03080 Alicante. SPAIN

(3) Departamento de Física Aplicada. Escuela Superior de Ingeniería Informática.

Universidad de Castilla-La Mancha. Avda. de España, s/n. E-02071 Albacete. SPAIN

E-mail: a.belendez@ua.es

\begin{abstract}
An approximate scheme for obtaining the period of a simple pendulum for large amplitude oscillations is analysed and discussed. When students express the exact frequency or the period of a simple pendulum as a function of the oscillation amplitude, and they are told to expand this function in a Taylor series, they always do so using the oscillation amplitude as the variable, without considering that if they change the variable (in this paper to the new variable $m$ ), a different Taylor series expansion may be done which is in addition more accurate than previously published ones. Students tend to believe that there is one and only one way of performing a Taylor series expansion of a specific function. The approximate analytical formula for the period is obtained by means of a Taylor expansion of the exact frequency taking into account the KiddFogg formula for the period. This approach based on the Taylor expansion of the frequency about a suitable value converges quickly even for large amplitudes. We believe that this method may be very useful for teaching undergraduate courses on classical mechanics and helping students understand nonlinear oscillations of a simple pendulum.
\end{abstract}

KEY WORDS: Nonlinear pendulum; Period; Approximate formula; Taylor series expansion. 
A. Beléndez, E. Arribas, A. Márquez, M. Ortuño and S. Gallego, "Approximate expressions for the period of a simple pendulum using Taylor series expansion”, European Journal of Physics 32, 1303-1310 (2011).

The simple pendulum is perhaps one of the nonlinear systems most widely studied and analysed [1]. It is the most popular textbook example of a nonlinear system and is studied not only in advanced and introductory university courses on classical mechanics but also in physics education journals. Many nonlinear phenomena in the real world are governed by pendulum-like differential equations $[1,2]$; thus the equation describing a simple pendulum is one of the most important differential equations in physics [3]. The nonlinear differential equation for a simple pendulum can be solved exactly and the period and periodic solution expressions involve the complete elliptic integral of the first kind and the Jacobi elliptic functions, respectively [2, 4, 5]. For this reason, several approximation schemes have been developed to investigate the situation for large amplitude oscillations of a simple pendulum, and several approximations for its largeangle period have been proposed (a summary of most of them can be found in [3, 6-10]) that differ in complexity and domains of validity: some are intuitive, others use ingenuous strategies, while yet others are based on relatively sophisticated procedures [11]. These approximate expressions for the period of a simple pendulum may be obtained in three ways, and in some cases the same results are reached. Firstly, analytical solutions for the period may be found using methods for approximately solving the nonlinear differential equation governing the behaviour of a simple pendulum, such as those based on perturbations, harmonic balance or cubing the differential equation. In all cases, the intention is to approximately solve the exact non-linear differential equation. Secondly, approximate analytical expressions for the period may be proposed, some of which are intuitive, some ingenious and yet others involve quite sophisticated procedures. All these may be called "heuristic approximations", since the approximate expression for the period is introduced "ad hoc". These include the well-known Kidd and Fogg expression and others published in this journal. Finally, the least common way is to obtain an approximate expression using the exact equation for the period of a simple pendulum which, as we know, is expressed in terms of a complete elliptic integral of the first kind. This is precisely the procedure followed in our paper to obtain an approximate analytical expression for the period of a simple pendulum in terms of elemental mathematical functions (in this case, mainly cosines and square roots). This approximate expression may in turn be considered accurate taking into account its simplicity. This in essence is the novelty of our article: we show that an accurate approximation, given its 
A. Beléndez, E. Arribas, A. Márquez, M. Ortuño and S. Gallego, "Approximate expressions for the period of a simple pendulum using Taylor series expansion”, European Journal of Physics 32, 1303-1310 (2011).

simplicity, may be obtained using an unsophisticated procedure (a Taylor series expansion).

Approaches based on the expansion of the period in terms of a power series of the oscillation amplitude $\theta_{0}$ or a power series of $\sin ^{2}\left(\theta_{0} / 2\right)$ are sometimes studied in intermediate courses at university [12], but converge slowly even for intermediate amplitudes [9]. In these approaches the integrand of the complete elliptic integral of the first kind is expanded in a Taylor series about $\theta_{0}=0$ or $\sin ^{2}\left(\theta_{0} / 2\right)=0$. One of the heuristic approximate expressions proposed for the period of a simple pendulum, $T$, the Kidd-Fogg formula [13], has attracted much interest due to its simplicity [14]. It is given by $T_{K F}=T_{0} / \sqrt{\cos \left(\theta_{0} / 2\right)}$, where $T_{0}$ is the period for small angle oscillations. Kidd and Fogg obtained their approximate expression making an analogy between the equation for the period of the pendulum for small oscillations and the period of a simple harmonic oscillator. Subsequently, some sophisticated reasoning have been used to justify the Kidd-Fogg expression. One of them more known was made by Millet [15], who justified the Kidd-Fogg formula taking into account the well-known trigonometric relation for the half-angle formula $\sin \theta=2 \sin (\theta / 2) \cos (\theta / 2)$ and considering that $\sin (\theta / 2)$ would have to be replaced by $\theta / 2$ to give a linear function, and $\cos (\theta / 2)$ would have to be replaced by $\cos \left(\theta_{0} / 2\right)$ to obtain a constant, so the standard approximation $\sin \theta \approx \theta$ could be improved to $\sin \theta \approx \theta \cos \left(\theta_{0} / 2\right)$. Then the nonlinear differential equation for the simple pendulum becomes a linear differential equation analogous to the one for the simple harmonic oscillator but with a frequency depending on the oscillation amplitude.

In this letter we derive a simple and accurate approximate formula for the period of a simple pendulum, which is analogous to that obtained by Carvalhaes and Suppes [11, 16] using reasoning based on the arithmetic-geometric mean. We obtain this expression taking into account the Kidd-Fogg formula and doing a Taylor expansion of the exact frequency. However, instead of doing the series expansion of the period about $\sin ^{2}\left(\theta_{0} / 2\right)=0$ (MacLaurin expansion) as a power series of $\sin ^{2}\left(\theta_{0} / 2\right)$, as one can find in all textbooks and journal papers, we propose to do a Taylor expansion of the exact frequency - written as a function of $\sqrt{\cos \left(\theta_{0} / 2\right)}$, which is the dimensionless Kidd-Fogg frequency- about $\sqrt{\cos \left(\theta_{0} / 2\right)}=1$. This will allow us, firstly, to obtain a simple, accurate approximate expression for the frequency (and thus for the period), and 
A. Beléndez, E. Arribas, A. Márquez, M. Ortuño and S. Gallego, "Approximate expressions for the period of a simple pendulum using Taylor series expansion”, European Journal of Physics 32, 1303-1310 (2011).

secondly, to justify mathematically the Kidd-Fogg approximate formula without using a sophisticated reasoning.

Thus, the novelty of the procedure described in this study resides in the fact that we obtain quite an accurate expression, which is also mathematically simple, for the period of a simple pendulum by performing a Taylor series expansion of the exact expression for the frequency of a simple pendulum. However, instead of using the amplitude of oscillation $\theta_{0}$ as the variable for the Taylor expansion, we use $\sqrt{\cos \left(\theta_{0} / 2\right)}$. This allows us to obtain an expression of high quality, considering its mathematical simplicity, and at the same time mathematically justify Kidd and Fogg's expression. This equation, considered previously as a heuristic approximation, is in fact a mathematical approximation which may be obtained from a Taylor series expansion of the exact frequency. In this way, this letter allows students to see how, using the same expression (in this case the exact frequency of a simple pendulum), approximations of varying accuracy may be obtained if the right variable is chosen to do this Taylor expansion.

The equation of motion of a simple pendulum is the following nonlinear differential equation [1]

$$
\frac{d^{2} \theta}{d t^{2}}+\frac{g}{l} \sin \theta=0
$$

where $\theta$ is the angular displacement, $t$ is the time, $l$ is the length of the pendulum and $g$ is the acceleration due to gravity. Figure 1 shows a simple pendulum and the notation used in this letter. As Eq. (1) is nonlinear the period of the pendulum $T$ (and therefore the frequency $\omega=2 \pi / T$ ) depends on the amplitude of oscillations $\theta_{0}$ and its exact expression is

$$
\frac{T}{T_{0}}=\frac{2 K(k)}{\pi}
$$

where

$$
k=\sin ^{2} \frac{\theta_{0}}{2}
$$

and $K(k)$ is the complete elliptic integral of the first kind defined as follows 
A. Beléndez, E. Arribas, A. Márquez, M. Ortuño and S. Gallego, "Approximate expressions for the period of a simple pendulum using Taylor series expansion", European Journal of Physics 32, 1303-1310 (2011).

$$
K(k)=\int_{0}^{\pi / 2} \frac{d \phi}{\sqrt{1-k \sin ^{2} \phi}}
$$

In equation (2) $T_{0}=2 \pi \sqrt{l / g}$ is the period for small-angle oscillations. Note that $T$ diverges as $k$ approaches one $\left(\theta_{0} \rightarrow \pi\right)$.

The exact angular frequency of the motion of the simple pendulum can be written as follows

$$
\frac{\omega}{\omega_{0}}=\frac{\pi}{2 K(k)}
$$

where $\omega_{0}=\sqrt{g / l}$ is the frequency for small-angle oscillations. It is easy to verify that $\omega / \omega_{0}$ is equal to 1 at $k=0$ and vanishes at $k=1$. As we pointed out previously, in 2002, Kidd and Fogg [13] proposed an approximate formula for the large-angle pendulum period. It is given by

$$
\frac{T_{K F}}{T_{0}}=\frac{1}{\sqrt{\cos \left(\theta_{0} / 2\right)}}
$$

which can be written in terms of the angular frequency as follows

$$
\frac{\omega_{K F}}{\omega_{0}}=\sqrt{\cos \left(\theta_{0} / 2\right)}
$$

Considering equation (6) as the starting point, some other authors have obtained better approximate expressions for the period of a simple pendulum $[6,17]$. To do this, they expressed $\sqrt{\cos \left(\theta_{0} / 2\right)}$ in equation (7) as a function of $k$ (equation (3)) and they write

$$
\frac{\omega_{K F}}{\omega_{0}}=(1-k)^{1 / 4}
$$


A. Beléndez, E. Arribas, A. Márquez, M. Ortuño and S. Gallego, "Approximate expressions for the period of a simple pendulum using Taylor series expansion”, European Journal of Physics 32, 1303-1310 (2011).

which is used to obtain more accurate expressions. In this letter, we do precisely the opposite; we express $k$ as a function of $m=\sqrt{\cos \left(\theta_{0} / 2\right)}$, which is the value for the approximate frequency proposed by Kidd and Fogg (equation (7)). Doing this we obtain

$$
\sin ^{2}\left(\theta_{0} / 2\right)=1-\left(\sqrt{\cos \left(\theta_{0} / 2\right)}\right)^{4} \quad \text { and } \quad k=1-m^{4}
$$

Substituting equation (9) into equation (7), the Kidd-Fogg approximation is simplified to

$$
\frac{\omega_{K F}}{\omega_{0}}=m
$$

The percentage relative error for this approximate formula is less than $1 \%$ for $\theta_{0} \leq 96^{\circ}$ $(m=0.818)$.

The exact equation for the frequency in equation (5) can also be written in terms of $m$ instead of $k$. In this way, equation (5) becomes

$$
\frac{\omega}{\omega_{0}}=\frac{\pi}{2 K\left(1-m^{4}\right)}
$$

which is equal to 1 at $m=1$ and vanishes at $m=0$. As we pointed out previously, one of the first ways to obtain an approximate expression for the period (or the frequency) of a simple pendulum is to do the Taylor series expansion of equation (2) (or equation (5)) about $k=0$ (the MacLaurin series expansion). This can be seen in Marion's book [12] where the following power-series expansion is obtained

$$
\frac{T}{T_{0}}=1+\frac{1}{4} k^{2}+\frac{27}{192} k^{4}+\ldots
$$


A. Beléndez, E. Arribas, A. Márquez, M. Ortuño and S. Gallego, "Approximate expressions for the period of a simple pendulum using Taylor series expansion", European Journal of Physics 32, 1303-1310 (2011). doi: $10.1088 / 0143-0807 / 32 / 5 / 018$

and we can obtain the following power-series expansion for the exact frequency

$$
\frac{\omega}{\omega_{0}}=1-\frac{1}{4} k^{2}-\frac{5}{64} k^{4}+\ldots
$$

In order to obtain a more accurate, simple, approximate equation for the period of the nonlinear pendulum, we propose using equation (11) and doing a Taylor series expansion of this equation about $m=1$. The result we obtain using the MATHEMATICA program is

$$
\frac{\omega}{\omega_{0}}=1+(m-1)+\frac{1}{4}(m-1)^{2}+\ldots=\frac{1}{4}(1+m)^{2}+\ldots
$$

which coincides with the Kidd-Fogg approximation for small values of $m$ (considering only the first two terms in the power-series expansion in equation (14))

$$
\left(\frac{\omega}{\omega_{0}}\right)_{m \text { small }}=1+(m-1)=m=\frac{\omega_{K F}}{\omega_{0}}
$$

Now we demonstrate that the Kidd-Fogg formula can be mathematically obtained from the exact equation of the period doing a suitable Taylor expansion. From equation (14) we obtain the following approximate expression for the frequency of the simple pendulum

$$
\frac{\omega_{a 1}}{\omega_{0}}=\frac{1}{4}(1+m)^{2}
$$

Which can be finally written as follows

$$
\frac{\omega_{a 1}}{\omega_{0}}=\frac{1}{4}\left(1+\sqrt{\cos \left(\theta_{0} / 2\right)}\right)^{2}
$$


A. Beléndez, E. Arribas, A. Márquez, M. Ortuño and S. Gallego, "Approximate expressions for the period of a simple pendulum using Taylor series expansion", European Journal of Physics 32, 1303-1310 (2011).

and for the period we can write

$$
\frac{T_{a 1}}{T_{0}}=\frac{4}{\left(1+\sqrt{\cos \left(\theta_{0} / 2\right)}\right)^{2}}
$$

This is a very simple, highly accurate expression and it is easy to verify that its maximum percentage relative error is less than $0.12 \%$ at an angle of $140^{\circ}$ and less than $1 \%$ for $\theta_{0} \leq$ $163^{\circ}(m=0.384)$. In Figure 2 we plotted, as a function of $m$, the exact frequency (equation (11)), the Kidd-Fogg frequency (equation (15)) and the frequency derived in this letter (equation (16)). Figure 3 shows the same as Figure 2, but now as a function of amplitude of oscillation. In Figure 2, we marked the lines for which the relative errors for the approximate frequencies are $1 \%, m=0.818\left(96^{\circ}\right)$ for the Kidd-Fogg approximation and $m=0.384\left(163^{\circ}\right)$ for the approximation obtained in this letter (equation (16)).

The MacLaurin series expansion of equation (18) is given as follows

$$
\frac{T_{a 1}}{T_{0}}=1+\frac{1}{16} \theta_{0}^{2}+\frac{11}{3072} \theta_{0}^{4}+\frac{173}{737280} \theta_{0}^{6}+\frac{22616}{1321205760} \theta_{0}^{8}+\ldots
$$

The power-series expansion for the exact period in equation (2) is given as follows

$$
\frac{T}{T_{0}}=1+\frac{1}{16} \theta_{0}^{2}+\frac{11}{3072} \theta_{0}^{4}+\frac{173}{737280} \theta_{0}^{6}+\frac{22931}{1321205760} \theta_{0}^{8}+\ldots
$$

As can be seen, the first four terms of the series in equations (19) and (20) are identical and the difference between the coefficients of the fifth terms (power of $\theta_{0}^{8}$ ) is only around $1.4 \%$. To obtain a relative error of $1 \%$ for $\theta_{0} \leq 163^{\circ}(m=0.384)$ using the power series expansion in equation (20), we need to consider the first twelve terms.

Equation (17) coincides with the expression obtained by Carvalhaes and Suppes $[11,16]$ who used the arithmetic-geometric mean in order to approximate the complete elliptic integral of the first kind. They obtained different accuracies for various iterations. These iterations were carried out using the Legendre form of the arithmetic-geometric 
A. Beléndez, E. Arribas, A. Márquez, M. Ortuño and S. Gallego, "Approximate expressions for the period of a simple pendulum using Taylor series expansion”, European Journal of Physics 32, 1303-1310 (2011).

mean [11]. After two iterations they obtained equation (17). However, we believe the procedure we use to obtain this equation is easier because we only use a Taylor series expansion, which is more familiar to undergraduate students than the concept of the arithmetic-geometric mean, and this Taylor series expansion can be done without difficulty with the help of the MATHEMATICA program.

We could obtain a more accurate approximate expression considering one term more in the power-series expansion considered in equation (14) and the resulting expression is

$$
\frac{\omega}{\omega_{0}}=1+(m-1)+\frac{1}{4}(m-1)^{2}-\frac{1}{64}(m-1)^{4}+\ldots=\frac{1}{4}(1+m)^{2}-\frac{1}{64}(m-1)^{4}+\ldots
$$

and substituting $m=\sqrt{\cos \left(\theta_{0} / 2\right)}$ in this equation, we have

$$
\frac{\omega_{a 2}}{\omega_{0}}=\frac{1}{4}\left(1+\sqrt{\cos \left(\theta_{0} / 2\right)}\right)^{2}-\frac{1}{64}\left(1-\sqrt{\cos \left(\theta_{0} / 2\right)}\right)^{4}
$$

and the new approximate period expression becomes

$$
\frac{T_{a 2}}{T_{0}}=\frac{64}{16\left[\left(1+\sqrt{\cos \left(\theta_{0} / 2\right)}\right)^{2}-\left(1-\sqrt{\cos \left(\theta_{0} / 2\right)}\right)^{4}\right]}
$$

Now using this equation the relative error for the period is as low as $0.013 \%$ at an angle of $140^{\circ}$ and lower than $1 \%$ for $\theta_{0} \leq 168^{\circ}(m=0.323)$. Its MacLaurin expansion is given as follows

$$
\frac{T_{a 2}}{T_{0}}=1+\frac{1}{16} \theta_{0}^{2}+\frac{11}{3072} \theta_{0}^{4}+\frac{173}{737280} \theta_{0}^{6}+\frac{22931}{1321205760} \theta_{0}^{8}+\ldots
$$


A. Beléndez, E. Arribas, A. Márquez, M. Ortuño and S. Gallego, "Approximate expressions for the period of a simple pendulum using Taylor series expansion”, European Journal of Physics 32, 1303-1310 (2011).

As can be seen, the first five terms in the series in equations (19) and (24) are now the same.

In summary, in this letter we combined the Kidd-Fogg approximate expression for the period of a simple pendulum with the Taylor expansion of exact frequency (which can be easily obtained using well known computer programs such as MATHEMATICA) to obtain a simple, highly accurate approximate expression for the period, which also allows us to justify the Kidd-Fogg approximation. When students express the exact frequency of a simple pendulum as a function of $\theta_{0}, \omega\left(\theta_{0}\right)$, and they are told to expand this function in a Taylor series, they always do so using $\theta_{0}$ as the variable, without considering that if they change the variable (in this paper to the new variable $m=$ $\left.\sqrt{\cos \left(\theta_{0} / 2\right)}\right)$, a different Taylor series expansion may be done which is in addition more accurate than previously published ones. Students tend to believe that there is one and only one way of performing a Taylor series expansion of a specific function. Finally, we believe that the method used in this letter to obtain the approximate large-angle period formula is easier to understand for students on intermediate physics courses than the method proposed by Carvalhaes and Suppes using the arithmetic-geometric mean.

\section{Acknowledgments}

This work was supported by the "Vicerrectorado de Tecnología e Innovación Educativa" of the University of Alicante, Spain (GITE-09006-UA). 
A. Beléndez, E. Arribas, A. Márquez, M. Ortuño and S. Gallego, "Approximate expressions for the period of a simple pendulum using Taylor series expansion”, European Journal of Physics 32, 1303-1310 (2011).

doi: 10.1088/0143-0807/32/5/018

\section{References}

[1] Baker G L and Blackburn J A 2005 The Pendulum: A Case Study in Physics (Oxford University Press: Oxford)

[2] Lima F M S 2008 Simple 'log formulae' for the pendulum motion valid for any amplitude Eur. J. Phys. 29 1091-8

[3] Johannessen K 2011 An anharmonic solution to the equation of motion for the simple pendulum Eur. J. Phys. 32 407-17

[4] Lima F M S 2010 Analytical study of the critical behavior of the nonlinear pendulum Am .J. Phys. 78 1146-51

[5] Ochs K 2011 A comprehensive analytical solution of the nonlinear pendulum Eur. J. Phys. 32 479-90

[6] Beléndez A, Rodes JJ, Beléndez T and Hernández A 2009 Approximation for a large-angle simple pendulum period Eur. J. Phys. 30 L25-8

[7] Turkyilmazoglu M 2010 Improvements in the approximate formulae for the period of the simple pendulum Eur. J. Phys. 31 1007-11

[8] Beléndez A, Rodes JJ, Beléndez T and Hernández A 2009 Reply to "Comments on Approximation for a large-angle simple pendulum period” Eur. J. Phys. 30 L83-86

[9] Johannessen K 2010 An approximate solution to the equation of motion for largeangle oscillations of the simple pendulum with initial velocity Eur. J. Phys. 31 $511-18$

[10] Lima F M S 2008 Simple log formula for the pendulum motion valid for any amplitude Eur. J. Phys. 29 1091-8

[11] Carvalhaes C G and Suppes P 2008 Approximation for the period of the simple pendulum based on the arithmetic-geometric mean Am. J. Phys 76 1150-4

[12] Beléndez A, Hernández A, Beléndez T, Neipp C and Márquez A 2007 Application of the homotopy perturbation method to the nonlinear pendulum Eur. J. Phys. 28 93-104

[13] Beléndez A, Hernández A, Márquez A, Beléndez A and Neipp C 2006 Analytical approximations for the period of a nonlinear pendulum Eur. J. Phys. 27 539-51 
A. Beléndez, E. Arribas, A. Márquez, M. Ortuño and S. Gallego, "Approximate expressions for the period of a simple pendulum using Taylor series expansion”, European Journal of Physics 32, 1303-1310 (2011).

doi: 10.1088/0143-0807/32/5/018

[14] Gimeno E and Beléndez A 2009 Rational-harmonic balancing approach to nonlinear phenomena governed by pendulum-like differential equations $Z$. Naturforsch. 64a, 1-8

[15] Amore P and Aranda A 2005 Improved Lindstedt-Poincaré method for the solution of nonlinear problems J. Sound. Vib. 283 1115-36

[16] Beléndez A, Álvarez ML, Fernández E and Pascual I 2009 Cubication of conservative nonlinear oscillators Eur. J. Phys. 30 973-81

[17] Beléndez A, Francés J, Ortuño M, Gallego S and Bernabeu JG 2010 Higher accurate approximate solutions for the simple pendulum in terms of elementary functions Eur. J. Phys. 31 L65-L70

[18] Marion J B 1970 Classical Dynamics of Particles and Systems (San Diego, CA: Harcourt Brace Jovanovich)

[19] Kidd R B and Fogg S L 2002 A simple formula for the large-angle pendulum period Phys. Teach. 40 81-3

[20] Lima F M S and Arun P 2006 An accurate formula for the period of a simple pendulum oscillating beyond the small angle Am. J. Phys. 74 892-5

[21] Millet L E 2003 The large-angle pendulum period Phys. Teach. 41 162-3

[22] Carvalhaes C G and Suppes P 2009 The high-precision computation of the period of the simple pendulum Rev. Bras. Ens. Phys $\mathbf{3 1}$ art2701

[23] Hite G E 2005 Approximations for the period of a simple pendulum Phys. Teach. $43290-2$ 
A. Beléndez, E. Arribas, A. Márquez, M. Ortuño and S. Gallego, "Approximate expressions for the period of a simple pendulum using Taylor series expansion", European Journal of Physics 32, 1303-1310 (2011).

doi: $10.1088 / 0143-0807 / 32 / 5 / 018$

\section{FIGURE CAPTIONS}

Figure 1.- Simple pendulum parameters.

Figure 2.- The exact frequency (equation (11)), the Kidd-Fogg frequency (equation (15)) and the frequency derived in this letter (equation (16)) as a function of $m=\sqrt{\cos \left(\theta_{0} / 2\right)}$. We marked the lines for which the relative error for the approximate frequencies is $1 \%: m=0.818\left(96^{\circ}\right)$ for the Kidd-Fogg approximation and $m=0.384\left(163^{\circ}\right)$ for the approximation derived in this letter (equation (16)).

Figure 3.- The exact frequency (equation (11)), the Kidd-Fogg frequency (equation (15)) and the frequency derived in this letter (equation (16)) as a function of amplitude of oscillation. 
A. Beléndez, E. Arribas, A. Márquez, M. Ortuño and S. Gallego, "Approximate expressions for the period of a simple pendulum using Taylor series expansion", European Journal of Physics 32, 1303-1310 (2011).

doi: $10.1088 / 0143-0807 / 32 / 5 / 018$

FIGURE 1

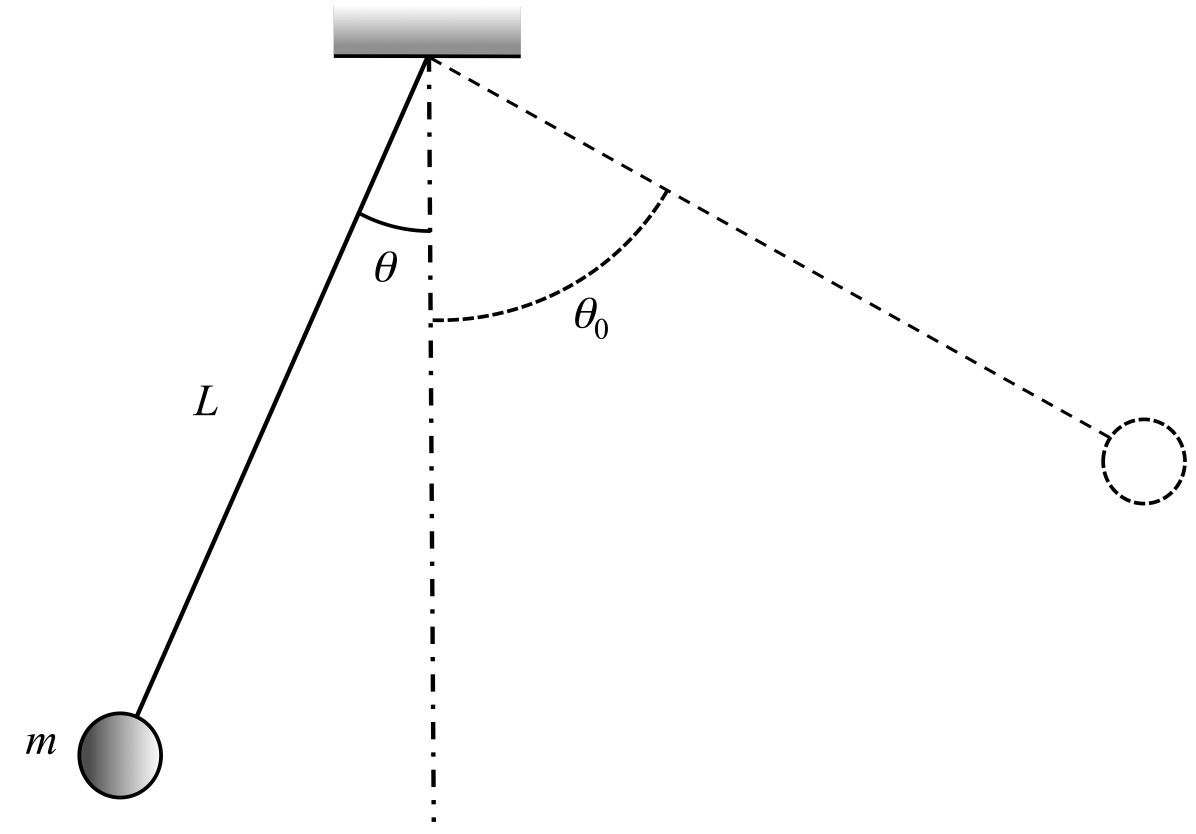


A. Beléndez, E. Arribas, A. Márquez, M. Ortuño and S. Gallego, "Approximate expressions for the period of a simple pendulum using Taylor series expansion”, European Journal of Physics 32, 1303-1310 (2011). doi: 10.1088/0143-0807/32/5/018

\section{FIGURE 2}

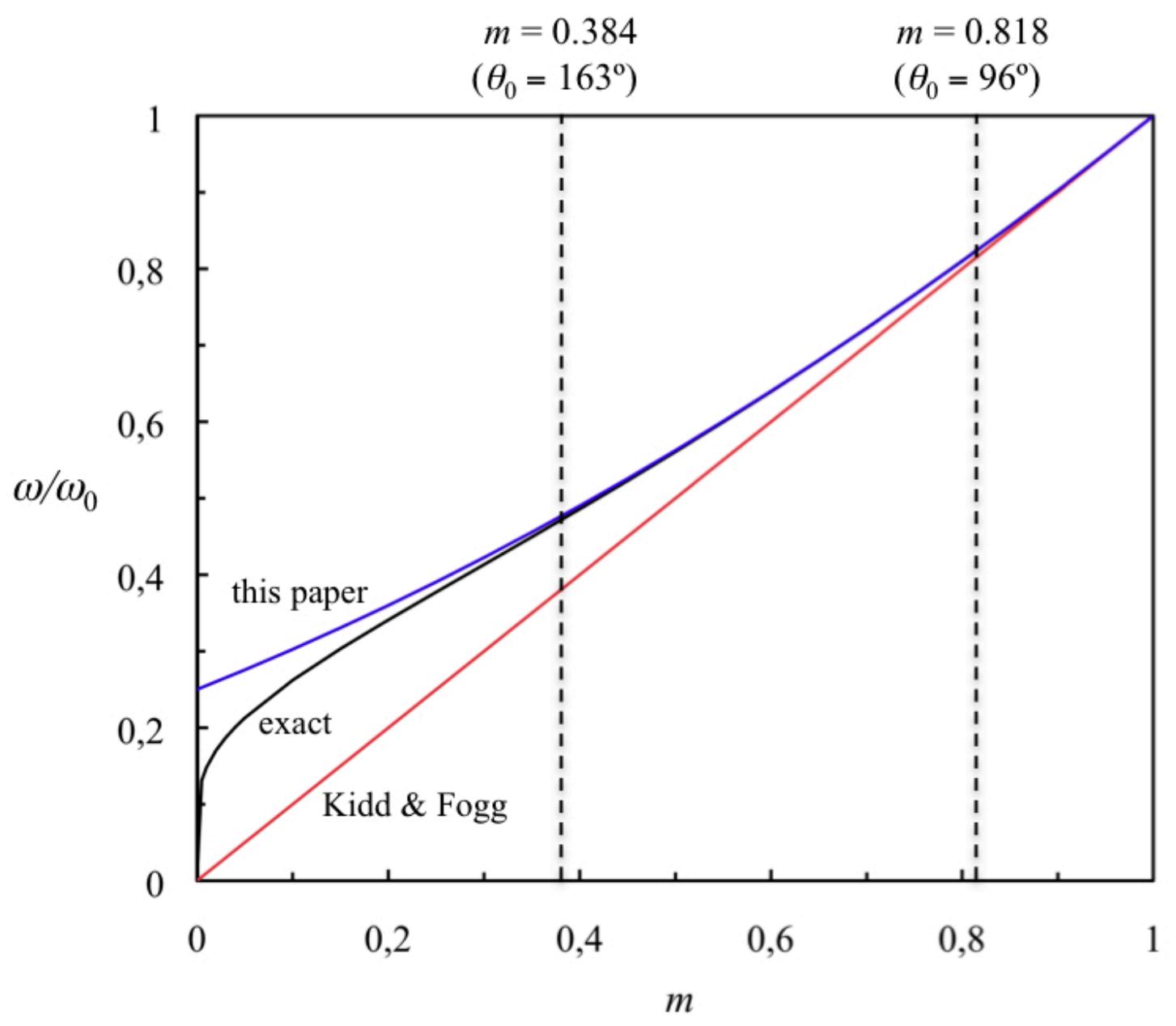


A. Beléndez, E. Arribas, A. Márquez, M. Ortuño and S. Gallego, "Approximate expressions for the period of a simple pendulum using Taylor series expansion", European Journal of Physics 32, 1303-1310 (2011). doi: 10.1088/0143-0807/32/5/018

\section{FIGURE 3}

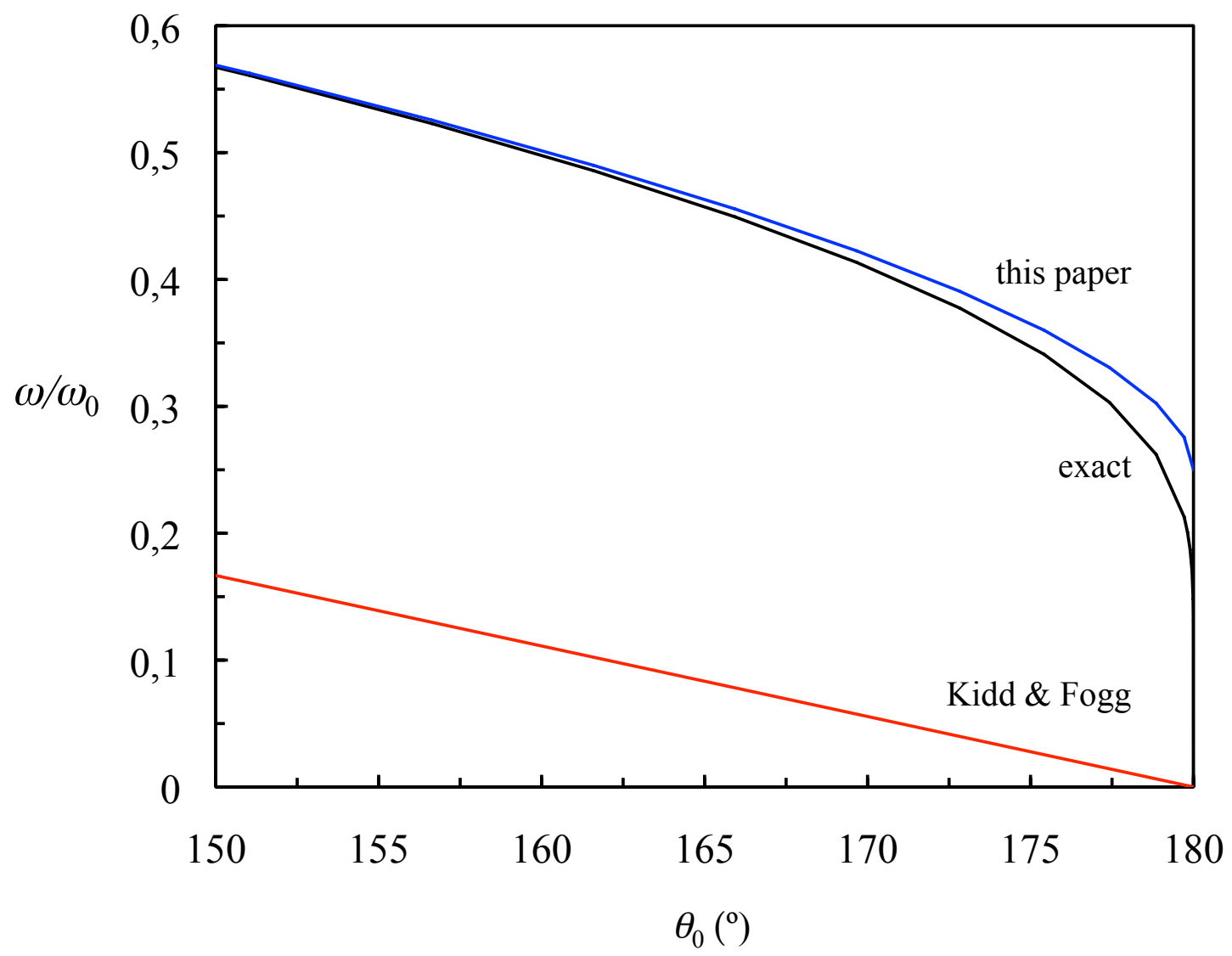

